\title{
Evangélicos en Bilbao: ser crevente en minoría
}

\section{Miren Iziar Basterretxea* Lidia Rodríguez* Luzio Uriarte*}

\section{Resumen}

El País Vasco, de tradición fuertemente católica, ha experimentado en los últimos años un proceso de "descreencia" que lo sitúa como uno de los territorios más distanciados de la religión de España. A ello se suma el hecho de que la distancia respecto a la religión es, además, combativa, es decir: no solamente casi el 50\% de la población se declara como "no creyente", sino que su actitud hacia la Iglesia Católica es claramente de rechazo. En este contexto, el resto de las religiones, cuyos porcentajes (siendo aún muy bajos) van en aumento, se sitúan en una posición de minoría no únicamente numérica, sino social. En el presente trabajo nos acercaremos a conocer hasta qué punto, en un contexto con una secularización tan acusada, esta posición de minoría afecta tanto a las creencias como a la forma de vivirlas de las personas protestantes en general y, específicamente, de las evangélicas. Realizado en Bilbao a partir de entrevistas en profundidad a evangélicos, tanto latinoamericanos como autóctonos, los primeros resultados apuntan a la autonomía interpretativa, a una valoración ambigüa de la diversidad y a la privatización de la vivencia religiosa como características más relevantes.

Palabras clave: evangélicos, latinoamericanos, autonomía, diversidad religiosa, Bilbao.

*Universidad de Deusto, Bilbao, España. 


\section{Evangelicals in Bilbao: being a minority believer}

\section{Abstract}

The Basque Country, which has a strong Catholic tradition, has experienced in recent years a process of "disbelief" that places it as one of the territories most distanced from religion in Spain. Added to this is the fact that such distance regarding religion is also combative, that is, not only almost $50 \%$ of the population declares themselves as "non-believers" but their attitude towards the Catholic church is clearly of rejection. In this context, the rest of the religions, whose percentages, being still very low, are increasing, are placed in a position of minority not only numerical but also social. In this paper we seek to understand to what extent, in a context of pronounced secularization, this minority position affects both the beliefs and the way of living of Protestant people in general and, specifically, evangelicals. Conducted by means of in-depth interviews with Evangelicals, both Latin American and indigenous, in Bilbao, the first results point to interpretive autonomy, an ambiguous assessment of diversity and the privatization of religious experience as the most relevant characteristics.

Keywords: evangelicals, Latin Americans, autonomy, religious diversity, Bilbao.

\section{Introducción}

- I texto que presentamos a continuación se sitúa en el marco del - proyecto Lived religion, financiado por la Templeton Foundation y _ dirigido por Gustavo Morello (Boston College). La investigación del equipo de Morello se articuló en torno a la cuestión de la vitalidad religiosa latinoamericana, intentando encontrar una explicación mejor para dicha vitalidad que la dada por las actuales teorías de la secularización o de la elección racional.

La aproximación al problema se hizo a partir de las aportaciones de Nancy Ammerman y se trabajó durante los años 2015-2018 en los contextos urbanos de Córdoba (Argentina), Montevideo (Uruguay) y Lima (Perú). Su investigación se centró en comprender los modos en que la experiencia religiosa y/o la espiritualidad se concretan en las prácticas ordinarias de la vida cotidiana. 
En nuestro caso, a lo largo de los tres últimos años hemos replicado la investigación en la Comunidad Autónoma Vasca (en adelante CAV), aunque a menor escala. Nuestra intención ha sido obtener una perspectiva comparada entre la religiosidad vivida de la población latinoamericana en sus lugares de origen y la de los inmigrantes latinoamericanos residentes en la CAV, a la que se añade la comparación con la vivencia religiosa de la población autóctona.

El cuestionario utilizado para la realización de las entrevistas y los códigos para su análisis han sido consensuados entre los equipos que realizaron el estudio en las tres ciudades latinoamericanas y tiene dos partes: la primera hace referencia a características sociodemográficas, en la segunda se pregunta por aspectos y ámbitos de vida en los que consideramos que la vivencia religiosa puede tener lugar (la familia, el grupo de amigos, el trabajo....). Dadas las características de este tipo de método, se espera, además, que la persona entrevistada aporte referencias a otros espacios o ámbitos de trascendencia no previstos por quien investiga.

La selección de los informantes se ha realizado mediante una muestra intencional, en la que se ha buscado variedad en función del género, la edad, el lugar de residencia dentro de la ciudad y el tipo de vinculación religiosa. Para garantizar el anonimato, en esta presentación se han cambiado todos los nombres de los participantes.

El artículo aquí presentado se organiza en tres grandes apartados. En primer lugar, introducimos las características de la Comunidad Autónoma Vasca (CAV); después, presentamos algunas manifestaciones del fenómeno religioso en la CAV mediante una breve reseña histórica, seguida de una perspectiva cuantitativa de la situación actual. Para terminar, nos centramos en el análisis de las 11 entrevistas realizadas en Bilbao a creyentes evangélicos. Dado el número de entrevistas y el tipo de análisis, la última parte del artículo presenta algunas reflexiones en torno a las cuestiones que han aparecido en las entrevistas, acompañadas de cuestiones que consideramos de interés para próximos estudios. 


\section{La comunidad autónoma de Euskadi}

Todo fenómeno religioso debe comprenderse e interpretarse dentro de un determinado contexto sociocultural, tanto en su vertiente institucional como en lo relativo a las experiencias individuales (Estrada, 2001; Lenoir, 2012; González-Anleo; López-Ruiz, 2017; Mardones, 2003). Esto no significa que las estructuras sociales y el contexto cultural determinen la aparición, las características, la evolución o la desaparición de un determinado fenómeno religioso, pero sí constituyen las condiciones de plausibilidad que lo hacen, ya sea viable, ya sea improbable (Berger, 2016). Las consecuencias de esta afirmación sociológica se muestran especialmente significativas en los períodos de profundas transformaciones sociales y culturales (Ward, 2005), como el contexto presente. A continuación, apuntaremos brevemente las características socioculturales que nos parecen más relevantes para comprender la evolución del fenómeno religioso en la sociedad vasca en su conjunto, así como su situación actual.

La CAV está situada en el norte de la península ibérica y es una de las 17 Comunidades Autónomas españolas. Considerada, junto con Cataluña y Galicia, una de las comunidades "históricas" por sus peculiaridades culturales, tuvo un primer Estatuto de Autonomía durante la Segunda República española (1931-1939), para pasar durante el franquismo (19391975) a una situación de represión política y cultural. Un año después de la promulgación de la actual Constitución española (1978), con la llegada de la democracia, la CAV recuperó también su Estatuto de Autonomía (1979), el cual le confiere amplias competencias en áreas tan importantes para el desarrollo económico, social y cultural como la educación o la recaudación de impuestos. A día de hoy, cuenta con una población de 2.188.017 habitantes (EUSTAT, 2019). Tiene dos lenguas oficiales, el euskera y el castellano, y está constituida por tres provincias, Araba, Bizkaia - cuya capital es Bilbao - y Gipuzkoa, siendo Vitoria-Gasteiz la capital administrativa de la Comunidad.

La CAV es un territorio con una población fundamentalmente urbana. Su economía está basada, sobre todo, en los sectores secundario y terciario. Su renta per cápita y el nivel de bienestar la sitúan en la media de los países europeos. Sin embargo, desde el punto de vista demográfico se enfrenta 
al reto del envejecimiento de su población. La CAV tiene una esperanza de vida de 80,4 años para los varones y 86,3 para las mujeres (EUSTAT, actualizado abril 2019) lo que la coloca en la parte alta del ranking europeo. La tasa de natalidad, por el contrario, es la segunda más baja de Europa, 7,32 nacimientos por mil habitantes en 2018 (Euskadi.eus, 2018), sólo por detrás de la de Italia $(7,6)$ y por debajo de la media española $(8,4)$. En estas condiciones, el incremento en números absolutos de su población se debe al flujo migratorio que actualmente representa el 10\% de la población (Ikuspegi, 2019) ${ }^{1}$ - de los cuales aproximadamente el 49,2\% proviene de América Latina y el Caribe, porcentaje que previsiblemente continuará incrementándose.

Bilbao, con 343.430 habitantes en su área municipal y aproximadamente seiscientos mil habitantes en su área metropolitana, es la ciudad más poblada de la CAV. Construida a orillas del río Ibaizabal y próxima al mar, desarrolló una pujante industria siderúrgica y naviera que posibilitó el desarrollo de una burguesía industrial, la cual apoyó numerosos proyectos sociales y culturales. En los últimos veinte años se ha redefinido como ciudad de servicios, con el museo Guggenheim como símbolo del cambio. Bilbao no sólo no es ajena al proceso demográfico señalado para la CAV, sino que su población de origen extranjero es incluso algo mayor que la media de la Comunidad y llega al 11,3\% (Ikuspegi, 2019)².

\section{El fenómeno religioso en la CAV}

\section{Breve reseña histórica}

Dadas las características de este trabajo, no vamos a extendernos en detallar las distintas creencias que han habitado lo que hoy es el territorio de la CAV, Comunidad Autónoma que se define administrativamente a

\footnotetext{
${ }^{1}$ Las páginas web de estas instituciones son: www.eustat.eus, www.ikuspegi.eus, www. euskadi.eus,

${ }^{2}$ Los datos de Ikuspegi (Observatorio Vasco de Inmigración) y los que ofrece el EUSTAT (Gabinete Estadístico del Gobierno Vasco) difieren ligeramente. El EUSTAT da unas cifras de población extranjera del 10,2\% en la CAV y del 12,2\% en Bilbao. Aquí, hemos optado por las cifras que arroja el estudio de Ikuspegi.
} 
partir de la Constitución española de 1978 y, específicamente, a partir de la Ley Orgánica 3/1979, de 18 de diciembre, de Estatuto de Autonomía para el País Vasco (BOE núm. 306, de 22 de diciembre de 1979).

No deja, sin embargo, de ser relevante recordar que lo que actualmente es un territorio del estado español fue, históricamente, lugar de convivencia de diferentes religiones, especialmente de tres: cristianismo, judaísmo e islam (Arregi; Canarias, 2010). La convivencia fue pacífica hasta, al menos, el siglo $\mathrm{XV}$, cuando judíos y musulmanes fueron obligados a optar por convertirse al cristianismo o ser expulsados del territorio español. En el siglo XVI hubo quienes, como el arzobispo de Toledo Bartolomé Carranza, simpatizaron con el pensamiento de la Reforma y llegaron a formarse núcleos de reformados, por ejemplo, en Valladolid o en Sevilla. Sin embargo, la intervención de la Inquisición y las condiciones históricas que habían comenzado en el s. $\mathrm{XV}$ impidieron que estas comunidades pervivieran. En el territorio vasco, la fe protestante tuvo el significativo apoyo de Juana de Albret, reina de Navarra, madre de Enrique IV de Francia y III de Navarra, que promovió la primera traducción de la Biblia al euskera.

Más próxima a nuestros días, la historia constitucional española fluctúa entre la confesionalidad y la laicidad. La Constitución de 1812 en su artículo $12^{3}$ y las de 1837 y 1845, ambas en sus respectivos artículos 11, declaran la religión "católica, apostólica y romana" como la religión del estado. El período entre 1939 y 1975 (fin de la guerra civil del 36 y muerte del dictador Francisco Franco) fue también un período de confesionalidad del estado. En un punto medio, la Constitución de 1869 (artículo 21) y la Constitución de 1876 (artículo 11) declaran que, si bien la religión de la nación es la católica, se reconoce la posibilidad de profesar otras religiones. Por último, declaradamente laicas, la Constitución de 1931 (artículos 3 y 26) y la actual constitución de 1978 en su artículo 16 proclaman que el estado español no tiene religión oficial.

El pluralismo religioso no es, pues, ajeno a la historia española. El reconocimiento constitucional del pluralismo no respondía únicamente al

\footnotetext{
${ }^{3}$ Artículo 12: "La religión de la Nación Española es y será perpetuamente la Católica [en mayúscula en el original], apostólica, romana, única verdadera. La Nación la protege por leyes sabias y justas y prohíbe el exercicio (sic) de cualquier otra". Todas las Constituciones españolas pueden consultarse en la página web del congreso español, www.congreso.es,
} 
deseo de las corrientes liberales de equipararse a las constituciones europeas más adelantadas en el reconocimiento de derechos: también respondía a la realidad social de algunos sectores, aunque pequeños, de la sociedad española del siglo XIX.

En la CAV, encontramos actualmente comunidades que representan las grandes familias de las iglesias protestantes, evangélicas y anglicana. Todas ellas surgen a finales del siglo XIX, fundadas por extranjeros que viajan por motivos laborales, especialmente relacionados con la actividad minera. Sin embargo, las comunidades que habían empezado a asentarse en el ambiente de libertad religiosa de la Segunda República se ven truncadas de nuevo por la represión franquista, régimen que prohíbe el culto y las manifestaciones públicas, expropia locales e incluso cementerios. Aún hoy, se da la situación de pastores o viudas de pastores que no pueden cobrar una pensión del estado, dado que en el franquismo no podían cotizar a la Seguridad Social al no estar legalmente reconocida su actividad (Rodríguez; Uriarte, 2010).

En los años finales del franquismo, a partir de 1965, se fundan en la CAV las primeras iglesias bautistas, grupos pentecostales y la llamada "Iglesia de Filadelfia", un movimiento autóctono con una gran aceptación entre el pueblo gitano. La presión internacional consiguió la aprobación de la ley 44/1971, la cual supuso un cierto reconocimiento legal, aunque siguió prohibiéndose la manifestación pública de la fe protestante.

Con la aprobación de la Constitución de 1978, que proclama la neutralidad religiosa del estado y la libertad de credo, pero sobre todo a partir de la aprobación de la Ley orgánica de libertad religiosa de 5 de julio de 1980, se constituye un marco normativo que equipara el reconocimiento de la libertad de conciencia y de religión con estándares internacionales y propicia la manifestación de la pluralidad religiosa. Aun así, persiste no sin polémica un tratamiento diferenciado hacia la Iglesia Católica a través del "concordato", un acuerdo entre el Estado y la Santa Sede con rango de convenio internacional. En cualquier caso, es a partir de los años 80 del siglo pasado cuando se establecen y desarrollan la mayoría de las actuales iglesias evangélicas y pentecostales presentes en la CAV. 


\section{La situación actual: el proceso en datos}

Los cambios sociales que hemos comentado han ido acompañados de transformaciones en la religiosidad de la sociedad vasca. Son fundamentalmente dos los procesos que afectan a la vivencia de la religión. Por una parte, como ya hemos apuntado, la llegada de inmigrantes a partir de los últimos años del siglo XX, que incrementa la diversidad religiosa. Por otra, durante los últimos años del franquismo y a lo largo de la etapa de la transición se produce un amplio y profundo proceso de secularización; los cambios que en otros países europeos se habían producido a lo largo de un siglo, en las sociedades vasca y española tienen lugar en una sola generación (Sáez-de-la-Fuente, 2007). Son años en los que España experimenta una "secularización más secularizada" (Aparicio; Tornos, 1995; Mardones, 2003; Sáez-de-la-Fuente, 2007; Pérez-Agote, 2009; 2012), proceso que supone la transición de un país de religión católica a otro de cultura católica.

Para dar una idea de hasta dónde han llegado ambos procesos, en este apartado se ofrecen algunos datos descriptivos de la situación actual, tanto en relación con la religiosidad y la diversidad religiosa en general, como en el caso de las iglesias evangélicas en particular. Tales datos no tienen pretensión de exhaustividad, no sólo porque no es el objetivo principal de este artículo, sino porque los estudios sobre estas cuestiones escasean (probablemente debido al mencionado alejamiento de la sociedad vasca respecto a la religión) y resulta difícil encontrar datos relacionados con la religión en las bases de datos de los organismos oficiales.

Hemos organizado los datos en dos apartados. En el primero presentamos algunos datos generales sobre religiosidad y otros datos específicos en relación con los evangélicos. En el segundo ofrecemos datos sobre la valoración que el pluralismo religioso merece, tanto en España como en la CAV. A la hora de leer estos datos debe tenerse en cuenta que los estudios no están elaborados a partir de la población total, sino con la información obtenida de muestras estadísticamente representativas de dicha población, y no de los subgrupos poblacionales que la conforman. 


\section{DATOS GENERALES DE RELIGIOSIDAD}

Las Tablas 1 y 2 muestran claramente el descenso de la religiosidad declarada en la CAV, al menos desde 1995. Aunque los datos provienen de estudios realizados a partir de una muestra poblacional, no deja de ser significativo que, de entre las opciones que propone la encuesta, la que mayor número concita es la de "ateos y agnósticos", seguida de "católicos no practicantes". También es importante el aumento de quienes declaran pertenecer a otras religiones

Tabla 1 - Autoadscripción religiosa

\begin{tabular}{|l|c|c|}
\hline \multicolumn{1}{|c|}{ Autodescripción } & $\mathbf{2 0 1 9}$ & \% \\
\hline Católico practicante & 307 & 14,3 \\
\hline Católico no practicante & 755 & 35,2 \\
\hline Otras Religiones & 147 & 6,9 \\
\hline Ateos y Agnósticos & 870 & 40,6 \\
\hline & $\mathbf{2 . 1 4 2}$ & $\mathbf{1 0 0}$ \\
\hline
\end{tabular}

Fuente: Elaboración propia a partir del Sociómetro Vasco. Junio, 2019.

Tabla 2 - Evolución de la autoadscripción religiosa (\%)

\begin{tabular}{|l|c|c|}
\hline \multicolumn{1}{|c|}{ Autodescripción } & $\mathbf{2 0 1 6}$ & $\mathbf{1 9 9 5}$ \\
\hline Católico practicante & 18 & 38 \\
\hline Católico no practicante & 37 & 44 \\
\hline Ateo y Agnóstico & 34 & 15 \\
\hline Otras religiones & 6 & 1 \\
\hline Dios si, religión no & 4 & 2 \\
\hline & $\mathbf{1 0 0}$ & $\mathbf{1 0 0}$ \\
\hline
\end{tabular}

Fuente: Elaboración propia a partir del Anuario de la opinión pública vasca 2016.

Sin embargo, como vemos en la tabla siguiente (Tabla 3), cuando preguntamos al grupo que se ha definido como "practicante" en cualquiera de las religiones "¿Con qué frecuencia va Ud. a la iglesia, u otro tipo de lugar de culto?", las respuestas marcan una ligera diferencia ascendente en la práctica diaria y en la de "más de una vez por semana". 
Tabla 3 - Asistencia al culto, entre quienes se declaran católicos practicantes o pertenecientes a otras religiones (\%)

\begin{tabular}{|l|c|c|}
\hline \multicolumn{1}{|c|}{ Frecuencia } & $\mathbf{2 0 1 6}$ & $\mathbf{1 9 9 5}$ \\
\hline Diariamente & 8 & 5 \\
\hline Más de 1 vez por semana & 12 & 11 \\
\hline 1 vez por semana & 41 & 56 \\
\hline Menos de 1 vez por semana & 31 & 27 \\
\hline
\end{tabular}

Fuente: Elaboración propia a partir del Anuario de la opinión pública vasca 2016.

Las dos tablas anteriores (Tablas 2 y 3 ) nos muestran un aspecto del que desconocemos su evolución posterior, pero que merece atención. En primer lugar, como viene siendo habitual en los datos evolutivos, el porcentaje de personas que se declara creyente católico disminuye, mientras que los porcentajes de las demás religiones crecen. Como se aprecia con claridad, quienes más aumentan son los ateos y agnósticos, cuyo peso en la población total se duplica. Junto a este proceso, parece apuntarse también un aumento en la práctica diaria de quienes se declaran "practicantes" de cualquiera de las religiones, dato que probablemente tiene que ver con el incremento de la diversidad religiosa.

La tabla siguiente (Tabla 4) nos muestra la posición de la comunidad evangélica dentro del grupo de religiones minoritarias en la CAV. La comunidad evangélica es la más numerosa y la que más centros o locales de culto tiene. Si tenemos en cuenta que estos datos corresponden a un estudio realizado hace 4 años, podemos suponer que el número de fieles en la actualidad se habrá incrementado. De hecho, según el informe Directorio de lugares de culto. Octubre 2019 del Observatorio del Pluralismo Religioso (2019) ${ }^{4}$, los lugares de culto evangélico en la CAV ascienden actualmente a 130.

Para terminar, a pesar del descenso en la adscripción religiosa, algunos estudios indican que, en tanto que fenómeno cultural, la adscripción religiosa sigue teniendo relevancia, por ejemplo, en el voto. La religiosidad es una variable que permite discriminar entre el Partido Socialista Obrero Español (PSOE, izquierda) y el Partido Popular (PP, derecha), especialmente en las

\footnotetext{
${ }^{4}$ Sítio de internet: www.observatorioreligion.es
} 
elecciones donde entran en juego cuestiones morales, como el divorcio o el aborto (Cordero, 2014).

Tabla 4 - Número de entidades y estimación del número de fieles de las religiones minoritarias - Euskadi

\begin{tabular}{|c|c|c|c|c|}
\hline Iglesias/Confesiones & Entidades & $\%$ Entidades & Fieles & \% Población \\
\hline Evangélicos & 122 & 41,5 & 19.500 & 0,90 \\
\hline Islam & 65 & 22,0 & 12.600 & 0,57 \\
\hline Testigos cristianos de Jehová & 63 & 21,5 & 4.800 & 0,22 \\
\hline Ortodoxos & 18 & 6,0 & 2.500 & 0,12 \\
\hline Budistas & 12 & 4,0 & 540 & 0,02 \\
\hline IJSUD - Mormones & 11 & 1,0 & 500 & 0,02 \\
\hline Otras & 4 & 4,0 & 2.300 & 0,10 \\
\hline TOTAL & 295 & 100 & 42.740 & 1,98 \\
\hline
\end{tabular}

Fuente: Urrutia-Asúa (2016, p. 134 y 153).

\section{VALORACIÓN DE LA DIVERSIDAD RELIGIOSA}

Para concluir esta mirada descriptiva a los aspectos cuantitativos, nos parece importante detenernos en conocer alguna de las actitudes y valoraciones que los vascos declaran frente a la cuestión de la diversidad religiosa, la cual se percibe como un fenómeno relacionado, fundamentalmente, con la inmigración (Tabla 5).

Tabla 5 - ¿Podría decirme cómo de extendida está en Euskadi la discriminación por... religión? (\%)

\begin{tabular}{|l|c|}
\hline Muy extendida & 6 \\
\hline Bastante extendida & 25 \\
\hline Poco extendida & 51 \\
\hline Nada extendida & 13 \\
\hline
\end{tabular}

Fuente: Anuario de la opinión pública vasca 2018 (Gobierno Vasco, 2019). 
Como puede observarse, la población de la CAV considera que la discriminación por religión está "poco o nada" extendida, respuestas que agrupan al $64 \%$ de la muestra. Además, a la pregunta "¿Está Ud. de acuerdo con... 'Las prácticas religiosas de algunas personas inmigrantes ponen en peligro nuestro estilo de vida'?", el 57\% de las personas se muestran en desacuerdo o muy en desacuerdo.

En España, el estudio no 3190 del CIS (2017), titulado Actitudes hacia la inmigración, constata una actitud similar en el conjunto de la población española. En este caso, la pregunta pedía valorar de 0 (muy negativo) a 10 (muy positivo) el hecho de que la sociedad española esté compuesta por personas de diferentes religiones. El 42,7\% de las respuestas estuvo entre el 7 y el 10. Al menos en las respuestas dadas conscientemente, no parece que la diversidad religiosa plantee problemas a la población, ni en el País Vasco, ni en el conjunto de España.

\section{La fe vivida por los evangélicos en Bilbao}

Como hemos señalado desde el principio, consideramos que la aportación principal de este artículo consiste en ofrecer una perspectiva desde la vivencia del creyente, en este caso evangélico. El enfoque de autoras como McGuire (2008) o Ammerman (2014) resalta la dinámica de la religión en la vida diaria de las personas, bajo la premisa de que ello no supone una renuncia a estudiar la religión, sino que más bien amplia dicho estudio.

Analizar todos los aspectos que aparecen en las entrevistas, si no imposible, sería una tarea que sobrepasaría los límites de este estudio, por lo que aquí presentaremos únicamente un acercamiento a las cuestiones relacionadas con la autonomía y con la diversidad religiosas. Ambos procesos han merecido la atención de diversos estudiosos (Berger, 1981; 2016; Davie, 1994; Mardones, 1994; Küng, 2004; Ferry; Gauchet, 2007; Beck, 2009; Levine, 2012; Pasquale; Kosmin, 2013; Duch, 2015; Bauman; Leoncini, 2018, entre otros). También en el ámbito de la CAV ha habido autores que han prestado atención al fenómeno como, por ejemplo, Ruiz-Vieytez (2010), Morondo-Taramundi y Ruiz Vieytez (2014), Rodríguez y Uriarte (2016), Urrutia-Asúa (2016), Elzo (2017), Uriarte y Urrutia-Asúa (2018). 
En las páginas que siguen presentamos las referencias a la autonomía y a la diversidad que aparecen en nuestro estudio, acompañadas de citas. Pero, antes de continuar, conviene apuntar, en primer lugar, que las personas entrevistadas no conceptualizan "autonomía" ni "diversidad"; estos conceptos son categorías que hemos establecido en el análisis de los textos de las entrevistas. En segundo lugar, las entrevistas realizadas a Evangélicos en Bilbao son pocas (11) y la aproximación es cualitativa, por lo que no arrojan resultados concretos, sino más bien indicadores que nos posibilitan establecer hipótesis para futuras investigaciones. En tercer lugar, hemos empleado el término "autóctono" para referirnos a las personas nacidas en la CAV, porque utilizar "local" nos parecía equívoco: quienes residen en Bilbao, aunque sean de origen latinoamericano, son población local. En cuarto lugar, dado que todas las entrevistas (tanto las realizadas en América Latina, en Roma, como en la (AV) se realizaron con el mismo guión y se analizaron con las mismas categorías, las comparaciones que puedan aparecer entre la población latinoamericana en Bilbao y en América Latina se hacen a partir de los resultados de los equipos de Córdoba, Uruguay y Perú (Morello, 2017; Morello et al., 2017; Rabbia et al., 2019). Por último, aunque no menos importante, todos los nombres que se mencionan son ficticios; se han cambiado para respetar el anonimato de las personas entrevistadas.

\section{La autonomía moral del sujeto}

Como hemos señalado, las personas entrevistadas no emplean la categoría "autonomía" para definir sus experiencias, pero a partir de lo que nos dicen podemos inferir lo que Rabbia et al. (2019) han denominado "marcadores de autonomía" o "peros", es decir: aquellas respuestas que comienzan explicando un "deber ser" para transformarse en un "ser" transgresor.

En el estudio latinoamericano se diferencian distintos niveles o aspectos en los que se manifiesta la autonomía. En primer lugar se encuentra la autoidentificación religiosa, que en Latinoamérica aparece en ocasiones como una identificación sui generis combinando clasificaciones consideradas 
a priori incompatibles, como por ejemplo "anarco-religiosa" (Rabbia et al., 2019). En Bilbao no hemos encontrado ninguna autoadscripción de este tipo.

En nuestro análisis tampoco aparece la autonomía respecto a las prácticas religiosas, es decir: los evangélicos de Bilbao, ya sean latinoamericanos o autóctonos, no mencionan otras prácticas más que las tradicionales, esto es, oración individual, asistencia al culto dominical y participación en tareas sociales de la comunidad. Estas últimas, si bien no son estrictamente prácticas religiosas "convencionales", han sido mencionadas de forma recurrente por los entrevistados. No se han mencionado, sin embargo, tatuajes, yoga etc. Tampoco aparece el uso con fines religiosos de las redes sociales.

Sí aparece, en cambio, la autonomía respecto a creencias o mandatos religiosos, más en concreto, en lo que podemos calificar de "cuestiones bioéticas".

Nieves, nicaragüense, nos cuenta que vivió

...una circunstancia muy dura de violencia de género [...] yo creo que Dios no nos ha mandado a someternos, por ejemplo, al yugo de la esclavitud de otra persona, y desde ese punto de vista es donde yo tomé la iniciativa de separarme, porque Dios dice en su palabra que él nos llamó a libertad.

Teresa, peruana, comparte sobre una chica de la que

...todo el mundo la ha apuntado con el dedo, inclusive mi iglesia, y al final me enteré que la chica decidió tenerlo [a su bebé], pero era una violación [...] En esa situación me pongo a pensar: "¿Qué tal si me hubiesen llegado a violar en ese asalto?" Yo hubiese abortado, te lo digo así, a mi libertad, te lo digo, a pesar de mis principios cristianos. [...] en lo de la homosexualidad, tengo dos amigos que son gays, no los juzgo [...] Sé que en mi religión eso no está bien visto, pero yo, en mi situación, en mi humilde opinión, como ser humana lo respeto.

Leire, de Bilbao, señala que

... hoy en día hay mucho homosexual, etcétera, ¿no? Esto la Biblia te dice que no está bien, pero bueno yo no voy a rechazar a una persona por eso, no se me ocurre. Entonces yo personalmente, mi pareja por ejemplo no lo ve así, pero yo sí. 
Por su parte, Yago, también de Bilbao, dice:

El que quiera ser homosexual que lo sea, igual en cuestión de lo que es el aborto, no sé, pues son niños que no tienen voz, entonces sí tienes que defender, pero el que tiene voz que se defienda él mismo y que decida lo que quiere ser.

Las dos primeras citas son de mujeres latinoamericanas que han vivido experiencias dolorosas, que han sentido miedo, que incluso refieren haber sentido el rechazo de su comunidad. Ambas mantienen su fe, pero reelaborada desde su libertad y su conciencia. Este tipo de experiencia constituye para nosotros lo que podríamos llamar "puntos de inflexión" en su itinerario vital, circunstancias que les hicieron replantearse y redefinir, no sin esfuerzo, sus posicionamientos individuales, la forma de integrar sus creencias en la vida y el compromiso que aquéllas conllevan. Dado que la vida siempre está abierta, los puntos de inflexión pueden sucederse, por lo que la fe está en constante "proceso", "en búsqueda" (Taylor, 2015). Nos preguntamos: estos procesos, ¿podrían considerarse igualmente parte de la reflexividad que caracteriza la modernidad? (Giddens, 1991).

Los dos últimos ejemplos muestran, de forma menos dramática pero también con claridad, el "pero" del que hablábamos en páginas anteriores. Leire se refiere tanto a la Biblia como a su pareja; sabe que tiene una perspectiva distinta sobre la homosexualidad, pero la asume. Mientras, Yago ni siquiera parece verlo como un problema, si bien no es tan explícito en su comentario.

Un último punto que nos parece interesante señalar es que ninguna de las personas entrevistadas ha respondido que acude al pastor cuando tiene problemas. Resulta llamativo, a pesar de que la fe evangélica tiene como punto de referencia central la obediencia a la Biblia y de que, como dice Viky, colombiana,

A mí no me rigen las normas, porque Dios me ha dado plena libertad. Soy una persona libre; lo que yo hago lo hago guiada por la Biblia, porque la palabra de Dios está por encima del pastor, yo no sirvo a los hombres. 
De nuevo, nos preguntamos: ipodría estar siendo redefinida la figura del pastor entre los evangélicos, tal y como sucede con la figura del presbítero entre los católicos, en tanto que figura de autoridad religiosa cada vez más institucionalizada y menos carismática?

\section{La diversidad religiosa}

En las entrevistas realizadas en Latinoamérica, las vivencias vinculadas a la diversidad religiosa se organizan en tres grupos, a saber: 1) quienes buscan individualmente y por diversos motivos el contacto con y el conocimiento de otras religiones; 2) quienes experimentan la diversidad a través de miembros de la familia extensa, los amigos o los conocidos; y 3) quienes conviven dentro de la familia nuclear con personas de otras creencias (Romero, 2019).

En las entrevistas realizadas en Bilbao no se menciona el primer tipo de diversidad. Nadie narra una historia, en la que busca intencionadamente encontrar la realización de su fe a través de la pertenencia seriada a confesiones o comunidades diferentes. Esto no significa que dicho fenómeno no se produzca en la CAV, pero en nuestro análisis no hemos encontrado ejemplos de ello. Sí hay, en cambio, experiencias de personas alejadas de la fe por circunstancias dramáticas de la vida que han conocido a alguien que les ha hablado de la fe evangélica, han entrado en contacto con comunidades evangélicas y han "conocido a Dios". Es el caso de Javier, que nos dice lo siguiente:

Mi testimonio es peculiar, porque yo estuve, yo tomaba drogas. Entonces fui a un centro de rehabilitación y ahí me ayudaron y me hablaron de que me acercase a Dios y ahí lo conocí, hace ya 30 años.

Respecto a la tercera forma de diversidad, la que se produce dentro de la familia nuclear, aparece solo en contadas ocasiones y de forma algo problemática. En las entrevistas se reflejan situaciones, en las que la familia de origen era de una creencia y la persona entrevistada ha cambiado; ésta no se siente comprendida, o incluso no aprueba lo que hace su familia, como Carol, quien comenta: 
Mi mamá, por ejemplo, ya tiene muchos años y ella sigue creyendo en sus vírgenes, y era un choque porque yo vivía en esa casa y yo no podía imponerme y decirles "tienes que tirar todas tus cosas". Tenía que aguantar y yo no... digamos esa parte, pero... me costaba, sí...

Con respecto a su madre, se trata de una diversidad inter-religiosa que no parece encajar muy bien. Carol menciona otra forma de diversidad intra-religiosa en referencia a su esposo:

...tenemos muchos puntos de vista que... inclusive en lo mismo si somos evangélicos, que a veces tienen diferentes puntos de vista, lo único que no estamos de acuerdo es que él no cree cuando te habla del Espíritu Santo.

Este tipo de diversidad no se entiende de forma positiva, porque genera conflictos entre personas a las que se respeta y con las que se tienen vínculos afectivos. A veces aparece la idea de que, felizmente, la diversidad inicial se ha superado por la conversión de la famila de origen, como nos dice Yago:

... mi hermana es la primera, luego fui yo y después mi otra hermana, y luego después de que murió mi padre ya mi madre también empezó a ir... Ahora más o menos sí, ya, todos.

El tercer ejemplo de diversidad que presentamos hace referencia al círculo más lejano de parientes y amigos. Aquí aparecen posturas contrapuestas: por una parte, están quienes ven la diversidad como una riqueza que permite el diálogo y el conocimiento; por otra, quienes manifiestan una postura más ambigua, donde no es fácil saber si se está reflejando una situación compleja, o bien se está valorando una opción como mejor que las demás. En el primer caso tenemos el testimonio de Nieves, nicaragüense, que tiene amigos católicos y musulmanes con los que habla "de temas espirituales, de temas sociales... de cómo es su dios, cómo es Alá". Comenta que le han invitado a comer por la noche durante el Ramadán, y añade: 
yo no pienso que haya una religión mala, la religión es un sistema creado por hombres bienintencionados para dar a conocer las verdades de Dios y del Evangelio a los seres humanos, pero esos hombres también cometen errores.

Con respecto a la segunda postura, David cuenta que

mis amigos pueden creer en Dios como creo yo. Muchos no son practicantes, muchos no están de acuerdo con unas cosas, muchos no están de acuerdo con otras cosas. Muchos han estado, se han desviado, se han ido. Sí que creo que, aunque creamos en lo mismo, todos somos diferentes.

A la vista de estos testimonios, nos parece que la cuestión de la diversidad religiosa aparece, cuanto menos, afectada por connotaciones ambiguas entre los evangélicos, ambigüedad a la que contribuye la multiplicidad de relaciones que rodean a las personas en las sociedades modernas.

Un aspecto de la diversidad que nos han manifestado algunos entrevistados tiene que ver con el contexto sociocultural de la sociedad de acogida. En la percepción de estas personas, la diversidad se sitúa en un marco de referencia más amplio, que incluye formas específicas de comportamiento. Este marco cultural influye, condiciona, limita, permite... distintas formas de diversidad, ya sea de género, de estatus socioeconómico etc., y entre esas varias formas de diversidad admitidas en la sociedad de acogida se encuentra la religiosa como una más. Mencionaremos únicamente dos contextos: aquél en el que las personas entrevistadas perciben la diversidad de forma positiva, y aquél en el que, por el contrario, se ve de una forma, si no negativa, al menos limitadora.

La valoración positiva es exclusiva de las mujeres. Nieves, a la que ya conocemos, dice así:

...aquí, la ventaja es que en este país hay muchísima ayuda para la mujer y allá no, allá existe una desigualdad tremenda, horrorosa... en mi iglesia no hay discriminación de género, todos participamos en la iglesia de la misma forma, como dirige un domingo un varón puede dirigir una mujer, predica un varón puede predicar una mujer.

Teresa, por su parte, comenta que 
...en mi país sí, las mujeres se van para un lado... y los hombres para otro lado [...] yo que estoy aquí viviendo, lo llamo machismo, porque otro nombre no puede tener, y aquí no, en la iglesia tanto las mujeres y los hombres opinamos por igual. De hecho, piden la opinión de la mujer cristiana y yo creo que es mucho más sabio pedir la opinión de la mujer cristiana.

La valoración no tan positiva es que la religión no se vive hacia fuera, sino hacia dentro, o al menos no se vive en la calle. Viky nos comenta que "en Colombia sí que se ve mucho el poder salir a evangelizar y hablar a las personas, aquí todavía falta mucho".

De nuevo, Teresa nos dice que

Cuando vamos de salida con amigos no cristianos nos acomodamos a ellos. A veces decimos: "¿Damos gracias por los alimentos?" Si nos dicen "si", oramos, y si nos dicen "no", no oramos. [...] Si se ve el momento idóneo, pues damos el punto de vista; si no, no.

De alguna manera, la vivencia religiosa de los evangélicos latinoamericanos en Bilbao se privatiza. Al contrario del contexto latinoamericano, donde la religión se expande y ocupa ámbitos no religiosos, las personas perciben con claridad que en Bilbao existen espacios vetados a la expresión de la vivencia religiosa. Esto no se percibe necesariamente como algo negativo; puede resultar complicado y quizá un tanto decepcionante, pero también aparece asociado a la idea del respeto mutuo. Nos parece importante hacer notar que las personas entrevistadas manifiestan sentirse respetadas en sus creencias, algo que parece concordar con lo visto en el apartado de datos cuantitativos al hablar de la valoración de la diversidad religiosa en la sociedad vasca. Viky comenta que "...la verdad es que aquí, en ese aspecto no cohíben a las personas, que esta religión o la otra, yo creo que en ese punto de momento lo están haciendo bien".

A modo de resumen, los comentarios que expresan las personas entrevistadas sobre la cuestión de la convivencia entre personas de creencias distintas parecen coincidir en que dicha convivencia debe basarse en el respeto mutuo y en la aceptación de ciertos límites al comportamiento en sociedad, también al comportamiento religioso. Por el momento, tales límites no se perciben como socialmente definidos, y las personas parecen 
ir estableciéndolos, dependiendo del contexto o de la situación concreta que viven.

\section{A modo de conclusiones}

Dado el carácter cualitativo y el número de entrevistas realizado, más que conclusiones en sentido estricto ofrecemos aquí algunas reflexiones que apuntan, no a certezas, sino a hipótesis que necesitan una posterior verificación.

En primer lugar, el análisis de las entrevistas indica que la creencia es "experiencial" y que se vincula a un claro proceso de autonomía. Al echar la vista atrás, la persona reflexiona sobre cómo ha ido reelaborando sus creencias y valores. En esta reelaboración, el contexto sociocultural y las experiencias vitales (sobre todo las más "traumáticas") se retroalimentan mutuamente, de forma que la norma religiosa queda matizada e incluso relativizada a partir de la interpretación de la experiencia, dando lugar a "puntos de inflexión" en la vida de los creyentes.

En segundo lugar, apreciamos que la diversidad religiosa se da tanto a nivel inter-religioso, como intra-religioso. En ambos casos se relaciona con las experiencias vitales de la persona y con sus esferas de relación y de afectividad. Para gestionar esta diversidad, valorada en las entrevistas de forma ambigua, aparece la idea de respeto mutuo, entendido como un compromiso tácito para aceptar ciertos límites a la hora de manifestar públicamente las creencias propias. Estos límites, sin embargo, no están prefijados y parecen tener que establecerse, al menos a la luz de la experiencia de los entrevistados, en cada situación concreta.

En tercer lugar, cabe preguntarnos hasta qué punto los procesos de autonomía y diversidad religiosa están impulsados por valores culturales que hoy se manifiestan de forma transversal en otras (y distintas) esferas de la vida social. En esta misma línea, deberíamos aclarar hasta qué punto las diferencias entre las posturas de los entrevistados que profesan creencias diversas son una mera cuestión de intensidad o matiz personal, o se deben a factores internos propios de cada tradición religiosa.

Por último, la gran pregunta que cabe hacerse tras el análisis de las entrevistas es si alguno de los procesos identificados tiene un carácter 
irreversible. Al parecer, las personas de distintas confesiones divergen menos entre sí, de lo que divergen las ortodoxias a las que se autoadscriben. El mismo hecho de la rediversificación religiosa en sociedades que creían haber resuelto "la cuestión religiosa" nos hace dudar de dicha irreversibilidad.

Miren Iziar Basterretxea es Doctora en Sociología y profesora de la Universidad de Deusto, España.

”ibaster@deusto.es.

Lidia Rodríguez es Doctora en Teología Bíblica y profesora de la Universidad de Deusto, España.

”. lidia.rodriguez@deusto.es.

Luzio Uriarte es Doctor en Teología y profesor de la Universidad de Deusto, España. ” luzio.uriarte@deusto.es.

\section{Referencias}

1. AMMERMAN, Nancy T. Sacred stories, spiritual tribes. Finding religion in everyday life. Nueva York: Oxford University Press, 2014.

2. APARICIO, Rosa; TORNOS, Andrés. ¿Quién es creyente en España hoy? Madrid: PPC, 1995.

3. ARREGI, Joxe; CANARIAS, Jorge. El País Vasco como encrucijada histórica de religiones. In: Ruiz-Vieytez, Eduardo J. (Dir.) Pluralidades latentes. Minorías religiosas en el País Vasco. Barcelona: Icaria Editorial, 2010, p. 61-80.

4. BAUMAN, Zigmund; LEONCINI, Thomas. Generación líquida. Transformaciones en la era 3.0. Barcelona: Paidós, 2018.

5. BECK, Ulrich. El dios personal: la individualización de la religión y el espíritu del cosmopolitismo. Barcelona: Paidós, 2009.

6. BERGER, Peter. Para una teoría sociológica de la religión. Barcelona: Kairós, 1981.

7. BERGER, Peter. Los numerosos altares de la modernidad. En busca de un paradigma para la religión en una época pluralista. Salamanca: Sígueme, 2016.

8. CIS - Centro de Investigaciones Sociológicas. Actitudes hacia la inmigración. Estudio 3190. CIS, 2017.

9. CORDERO, Guillermo. La activación del voto religioso en España (1979-2011). Revista Española de Investigaciones Sociológicas, n. 147, p. 3-20, 2014. 
10. DAVIE, Grace. Religion in Britain since 1945: believing without belonging. Oxford: Blackwell, 1994.

11. DUCH, Lluís. Antropología de la ciudad. Barcelona: Herder, 2015.

12. ELZO, Francisco J. Morir para renacer. Otra Iglesia posible en la era global y plural. Madrid: Ed. San Pablo, 2017.

13. ESTRADA, Juan A. Razones y sinrazones de la creencia religiosa. Madrid: Trotta, 2001.

14. EUSKADI.EUS. Indicadores municipales de sostenibilidad: tasa bruta de natalidad, 2018. Disponible en: https://www.euskadi.eus/indicadoresmunicipales-de-sostenibilidad-tasa-bruta-de-natalidad-x2030/web01-a2ingair/es/

15. EUSTAT - Instituto Vasco de Estadística. Estadística municipal de habitantes. Octubre, 2019. Disponible en: https://es.eustat.eus/estadisticas/tema_268/opt_1/ temas.html

16. FERRY, Luc.; GAUCHET, Marcel. Lo religioso después de la religión. Barcelona: Anthropos, 2007.

17. GIDDENS, Anthony. Modernity and self-identity. Self and identity in the late modern age. Stanford-California: Stanford University Press, 1991.

18. GOBIERNO VASCO. Gabinete de Prospección Sociológica. Anuario de la opinión publica vasca, 2018. Vitoria Gasteiz: Gobierno Vasco, 2019.

19. GONZÁLEZ-ANLEO, Juan; LÓPEZ-RUIZ, Jose A. Jóvenes españoles entre dos siglos 1984-2017. Madrid: Fundación SM, 2017.

20. IKUSPEGI. Observatorio Vasco de Inmigración. Evolución de la población total y de origen extranjero en la CAE y territorios históricos. 1998-2019, Ikuspegi, 2019. Disponible en: https://www.ikuspegi.eus/es/estadisticas/estadisticas.php

21. KÜNG, Hans. El nuevo paradigma de las relaciones internacionales y el papel de las religiones. In: TAMAYO, Juan J.; FORNET-BETANCOURT, Raul (Eds.) Interculturalidad, diálogo interreligioso y liberación. Estella: Verbo Divino, 2004, p. 17-30.

22. LENOIR, Frederic. Dios. Barcelona: Kairos, 2012.

23. LEVINE, Daniel H. Politics, religion and society in Latin America. Londres: Lynne Rienner Publishers, 2012.

24. MARDONES, Jose Maria. Para comprender las nuevas formas de la religión. La reconfiguración postcristiana de la religión. Estella: Verbo Divino, 1994.

25. MARDONES Jose Maria. La indiferencia religiosa en España ¿Qué futuro tiene el cristianismo? Madrid: HOAC, 2003.

26. MCGUIRE, Meredith. Lived religion. Faith and practice in everyday life. Nueva York: Oxford University Press, 2008. 
27. MORELLO, Gustavo. Modernidad y religiosidad en América Latina. Razón y Fe, v. 276, n. 1492, p. 327-338, 2017.

28. MORELLO, Gustavo; ROMERO, Catalina; RABBIA, Hugo; DA COSTA, Néstor. An enchanted modernity: making sense of Latin America's religious landscape. Critical Research on Religion, v. 5, n. 3, p. 308-326, 2017.

29. MORONDO-TARAMUNDI, Dolores; RUIZ-VIEYTEZ, Eduardo (Eds.). Diversidad religiosa, integración social y acomodos. Un análisis desde la realidad local en el caso vasco. Bruselas: P.I.E. Peter Lang, 2014.

30. OBSERVATORIO DEL PLURALISMO RELIGIOSO. Directorio de lugares de culto. Octubre 2019. Disponible en: http://www.observatorioreligion.es/ directorio-lugares-de-culto/

31. PASQUALE, Frank; KOSMIN, Barry. Atheism and the secularization thesis. In: BULLIVANT, Stephen; RUSE, Michael. The Oxford handbook of atheism. Oxford: Oxford University Press, 2013, p. 451-467.

32. PÉREZ-AGOTE, Alfonso. La secularización de los españoles. In: PÉREZAGOTE, Alfonso; GARCÍA, José Antonio. Religión y política en la sociedad actual. Madrid: CIS, 2009.

33. PÉREZ-AGOTE, Alfonso. Cambio religioso en España: los avatares de la secularización. Madrid: CIS, 2012.

34. RABBIA, Hugo $\mathrm{H}$. et al. La religión como experiencia cotidiana: creencias, prácticas y narrativas espirituales en Sudamérica. Lima: Fondo Editorial PUCP, 2019.

35. RODRÍGUEZ, Lidia; URIARTE, Luzio. El cristianismo reformado y evangélico. In: RUIZ-VIEYTEZ, Eduardo. Pluralidades latentes. Minorías religiosas en el País Vasco. Barcelona: Icaria Editorial, 2010. p 96-150.

36. RODRÍGUEZ, Lidia; URIARTE, Luzio. Religious minorities and access to public space in the Autonomous Community of the Basque Country and in Navarre: the perspective of religious minorities. In: WINKLER, Ulrich; RODRÍGUEZ, Lídia; LEIRVIK, Oddbjørn (Eds.). Contested spaces, common ground. Leiden: Brill, 2016. p. 243-252.

37. ROMERO, Catalina. Católicos en Lima vivendo encuentros interreligiosos. In: $\mathrm{RABBI}$, Hugo et al. La religión como experiencia cotidiana: creencias, prácticas y narrativas espirituales en Sudamérica. Lima: Fondo Editorial PUCP, 2019. p. 83-92.

38. RUIZ-VIEYTEZ, Eduardo. Pluralidades latentes. Minorías religiosas en el País Vasco. Barcelona: Icaria Editorial, 2010.

39. SÁEZ-DE-LA-FUENTE, Izaskun. Temas objeto de disputa en los debates sobre la laicidad. Enfoque sociopolítico. In: BILBAO, Galo; ETXEBERRIA, Xabier; ETXEBERRIA, Juan José; SÁEZ-DE-LA-FUENTE, Izaskun. La laicidad en los nuevos contextos sociales. Estudio interdisciplinar. Santander: Sal Terrae, 2007. p.15100. 
40. TAYLOR, Charles. La era secular. Barcelona: Gedisa, 2015.

41. URIARTE, Luzio; URRUTIA-ASÚA, Gorka. Minorías religiosas y centros de culto: percepción social y problemáticas para la gestión pública. Zerbitzuan: Revista de Servicios Sociales, v. 65, p. 53-64, 2018.

42. URRUTIA-ASÚA, Gorka. Minorías religiosas y derechos humanos. Reconocimiento social y gestión pública del pluralismo religioso en el País Vasco. Madrid: Akal, 2016.

43. WARD, Graham. Cultural transformation and religious practice. Cambridge: Cambridge University Press, 2005. 